$132 / 16 / 19$

LA-7876-MS

Informal Report

Homomorphic Processing of Seismic Signals

范

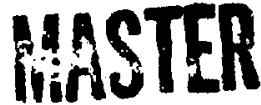

to

$\frac{7}{\infty}$

文 
LA-7876-MS

Informal Report

UC.34

Issued: June 1979

\title{
Homomorphic Processing of Seismic Signals
}

\author{
John E. Brolley
}

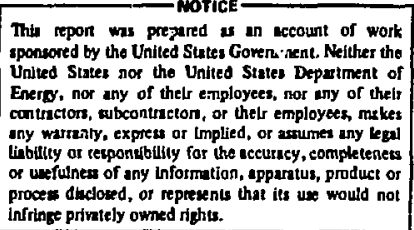


HOMOMORPHIC PROCESSING OF SEISMIC SIGNALS

by

John E. Brolley

ABSTRACT

An introduction to homomorphic processing of time series is presented. The basic concept is discussed and one form of implementation is adduced. Simple examples of reverberation problems are illustrated. Simple applications to real-time series associated with underground nuclear explosions and earthquakes are then presented.

\section{INTRODUCTION}

Determination of a physical descriptor for an underground reiease of energy is of considerable practical interest. One mode of attack uses seismometer signals. In this mode the associated seismic signals may be tracked in either direction. The present and ensuing reports will be concerned with the inverse problem tracing the signal back from the seismometer to the source.

A model suitable for didactic purposes is the following. The local energy release from the underground explosion or earthquake is described in terms of the earth motion near the energy release. Thus, there is some function $x(r, t)$, which we seek to find, that describes motion for location $r$ and time $t$ outside a region of inelastic behavior. It is possible to write a wave equation for $x(r, t)$ that is obeyed outside the inelastic zone. Analogous to electromagnetic theory, a scalar potential ${ }^{1-3}$ can be introduced to solve the wave equation for the body or compressional waves. For the case of the underground explosion it is possible to parametrize this potential in terms of explosion characteristics. The term $x(r, t)$ has a simple relation ${ }^{2}$ to the potential $\Phi$. The fourier transform of $\Phi(r, t)$ will be called the source function, $F(\omega)$, for this discussion. 
The source wavelet emitted by the explesion will propagate through the crust in which the explosion occurred, through the mantle and then through the crust again to a seismometer that has a characteristic response function. Each of these factors will convolve with the source wavelet to produce the seismometer time series. The Fourier transform, $S(\omega)$ of the time series will yield the product of the Fourier transforms of the convolution factors. ${ }^{4}$ Thus,

$$
S(\omega)=F(\omega) \cdot T(\omega) \cdot M(\omega) \cdot U(\omega) \cdot I(\omega),
$$

where $T(\omega)$ is the first crustal transfer function, $M(\omega)$ is the mantle transfer, $U(\omega)$ is the second crustal transfer function, and $I(\omega)$ is the instrument trans fer function. A gecmetrical factor has been ignored.

The objective is to recover a useful estimate of $F(\omega)$ from the data $S(\omega)$. The problem has already been simplified somewhat by transforming from a convolutional space to a product space.

\section{HOMOMORPHIC DECONVOLUTION}

$s(\omega)$ is a complex number resulting from taking the Fourier transform of a real time series $s(t)$. Oppenheim ${ }^{5}$ recognized that the product space of Eq. (1) could be transformed to an additive linear space by taking the complex logarithm of $S(\omega)$. Thus

$$
\log S(\omega)=\log F(\omega)+\log T(\omega)+\log M(\omega)+\log U(\omega)+\log I(\omega) .
$$

It is attractive to operate in this type of space. One notes that if estimates of the logarithms of $T(\omega), M(\omega), U(\omega)$, and $I(\omega)$ are available, they may be subtracted from $\log S(\omega)$ leaving only $\log F(\omega)$. It is often more convenient to have this linear space be in a field of real variables. This is accomplished by taking the inverse Fourier transform of Eq. (2). The result is a sequence of real numbers termed the complex cepstrum. Filtering can then be performed in the real domain and then the inverse operation performed. The sequence of operations is shown Figs. 1 and 2. This is 0ppenheim's procedure for homomorphic deconvolution.

The complex cepstrum is a sequence of real numbers. The appellation complex is employed to distinguish it from the cepstrum of Bogert et al. ${ }^{6}$ The complex cepstrum retains phase information while the other does not. 


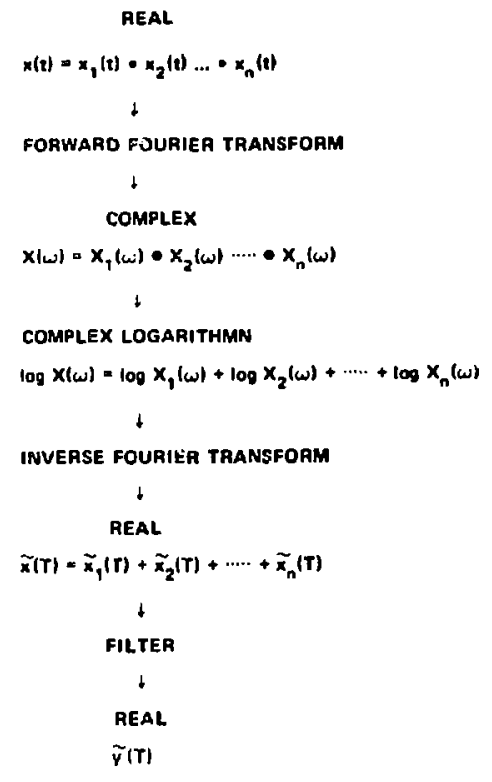

Fig. 1.

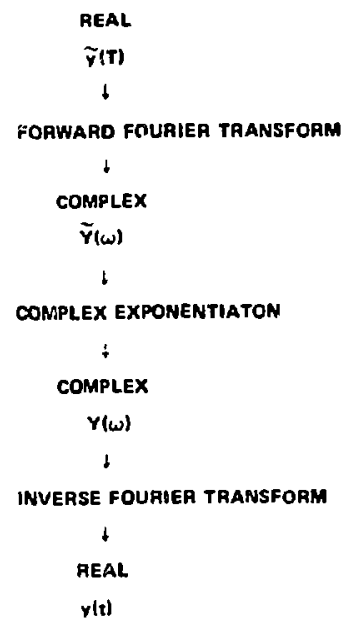

Fig. 2.

Computation of the complex cepstrum.

Regeneration from the complex cepstrum

\section{IMPLEMENTATION}

The most difficult step in the calculation of the complex cepstrum is the evaluation of the logarithm. This is a complex number that can be characterized by a magnitude and a phase. The problem can be defined in terms of properties of the z-transform of the original time series, the seismometer data. A time series, $x(t)$, can be characterized by its two-sided Z-transform,

$$
x(z)=\sum_{n=-\infty}^{\infty} x\left(t_{n}\right) z^{-n} .
$$

It can be shown that Eq. (3) may be written in terms of products of polynomials in $z$ having zeroes inside and outside the unit circle of the complex plain. For data sequenciss having zeroes near the unit circle, the calculation of the phase of logarithm becomes rather troublesome. Several methods ${ }^{7,8}$ have been employed. The present tutorial will use the procedure of Tribolet $^{8}$ that involves adaptive phase unwrapping.

If a time series has all of its zeroes, as defined above, outside the unit circle it is called a maximum phase signal. If all of its zeroes are inside, it is called a minimum phase signal. If it has zeroes both inside and outside, 
it is called a mixed-phase signal. It can be shown that the complex cepstra of minimum phase signals all reside in the positive time region, and the complex cepstra of maximum phase signals are in the negative time region. In general, source wavelets of interest will be mixed phase.

The convolution of the source function with the remaining factors may be thought of as convolving with an impulse function of the earth. ${ }^{9-11}$ It has been shown by Schafer ${ }^{2}$ that the impulse train can be made minimum phase by weighting the seismometer $t$ ime series with the exponential factor $\alpha^{\left(t_{n} / \Delta t\right)}$, where $\alpha<1$, and $\Delta t$ is the increment between data points. Further it can be shown that the source function will have most of its eriergy in the low time region ${ }^{9-11}$ of the complex cepstrum while the minimum phase impulse contribution will be in the high time region. Thus, by setting the complex cepstrum to zero above some low time value and performing the inverse operations, an estimate of the source function can be obtained. Zeroing the complex cepstrum on the other side of the low time value and performing the inverse operations will yield an estimate of the impulse train.

IV. EXAMPLES

For purposes of orientation, two examples ${ }^{12}$ which have known analytical solutions and two examples from real world time series will be given. A twopoint time series may be specified $t y$ the relation

$$
\begin{aligned}
& b^{t}[\delta(t)-\delta(t-\tau)] \\
& b=0.94
\end{aligned}
$$

It is plotted in Fig. 3. The complex cepstrum is given by

$$
\sum_{m=1}^{\infty} \frac{b^{m \tau}}{m} \delta(T-m \tau) .
$$

This is plotted in Fig. 4. The machine calculation, performed on a CDC 7600 , using the adaptive integration scheme, of the complex cepstrum is shown in Fig. 5. The regenerated time series obtained by performing a machine inversion of the cepstrum of Fig. 5 is shown in Fig. 6. Another time series is given by 


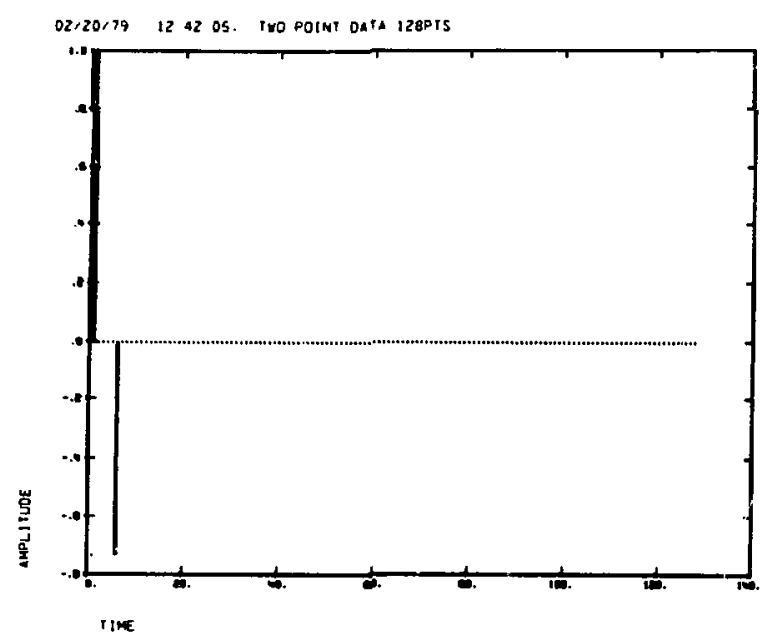

Fig. 3.

Two-point time series.

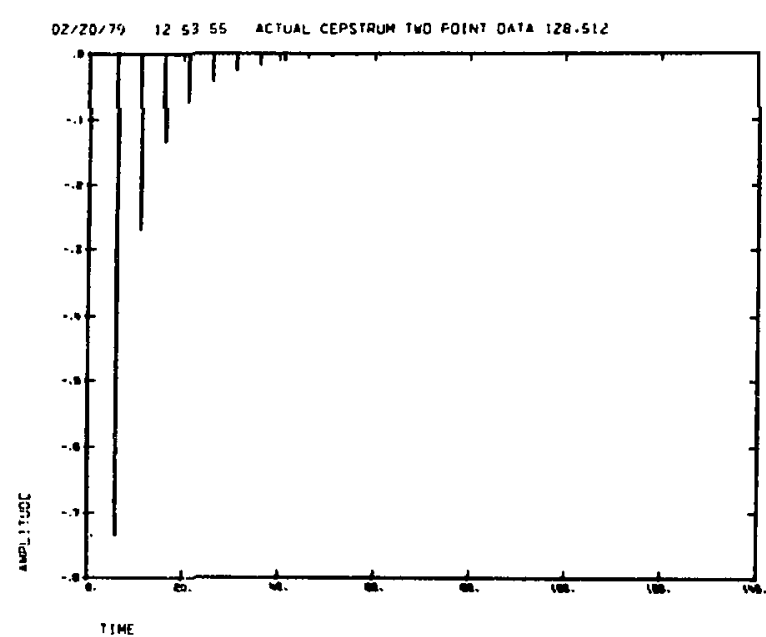

Fig. 4.

Exact complex cepstrum of the twopoint time series.

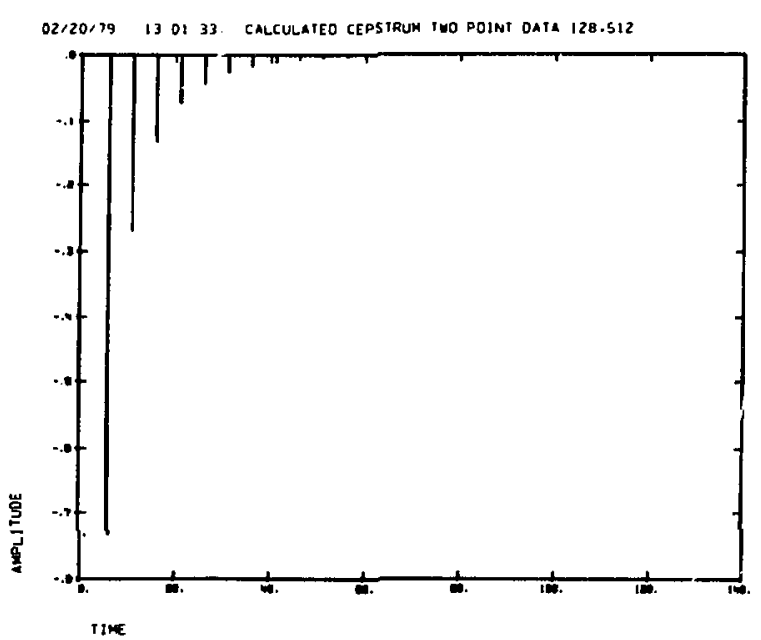

Fig. 5.

Machine calculation of the two-point time series complex cepstrum.

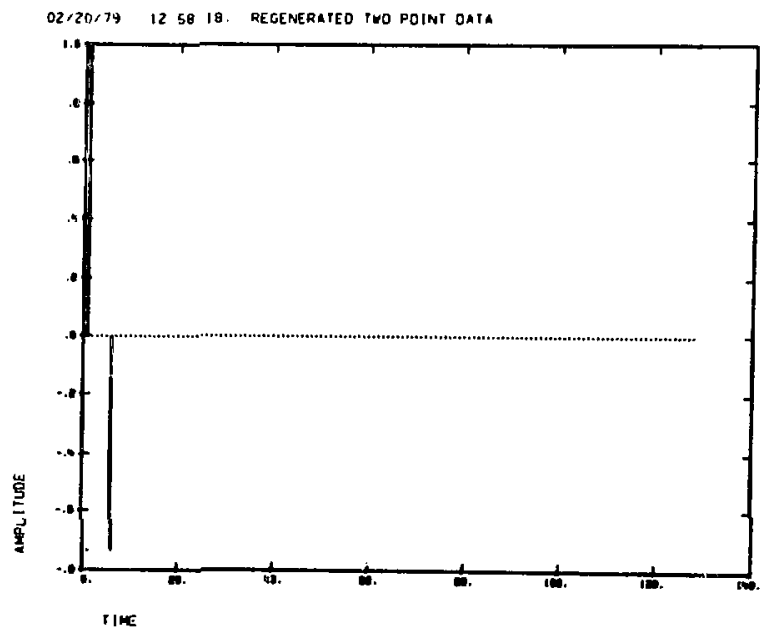

Fig. 6 .

Regeneration of the two-point time series from the machine calculation of the complex cepstrum. 


$$
\begin{gathered}
\sum_{n=0}^{\infty}(-1)^{n} R^{n} \delta(t-n \tau) \\
R=0.75
\end{gathered}
$$

This is plotted in Fig. 7. The complex cepstrum is given by

$$
\sum_{m=1}^{\infty}(-1)^{m} \frac{p^{m}}{m} \delta(T-m \tau)
$$

This is plotted in Fig. 8. The corresponding machine calculation is shown in Fig. 9. The machine regenerated time series is shown in Fig. 10. Both of these time series are minimum phase.

Next a seismogram from the Albuquerque Seismic Research Observatory (SRO) station of the North China earthquake of 27 July 1976 is shown in Fig 11. This is the first portion of the short period (20 samples per second) record. The initial portion of Fig. 10 is displayed in more de tail in Fig. 12. In this and the following example, no attempt has been made to precisely identify the arrival time. An exponential weighting factor, $a=0.94$, was applied to the time

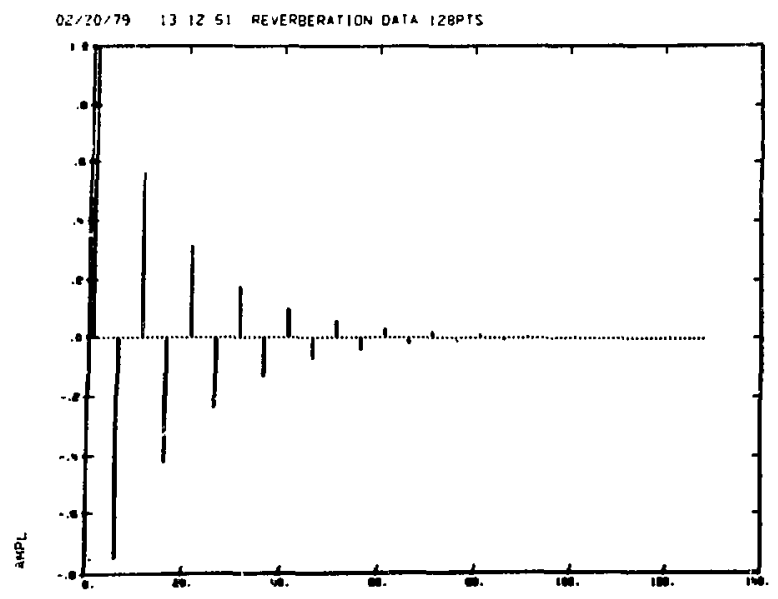

Fig. 7. Time series that models a simple reverberation problem.

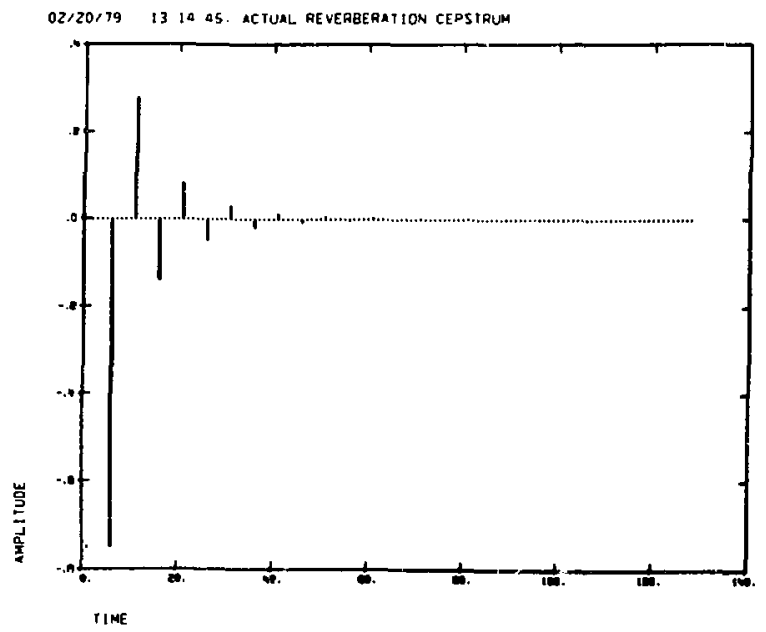

Fig. 8.

Exact complex cepstrum of Fig. 7 . 


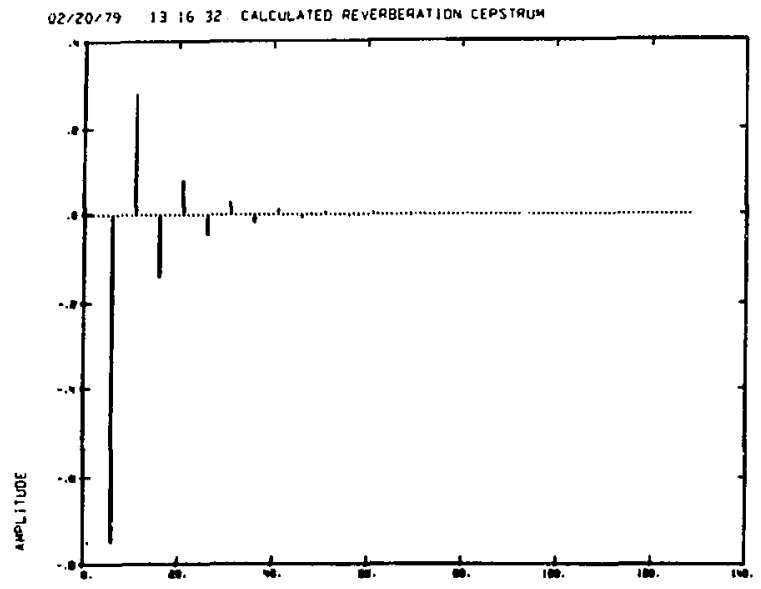

TIME

Fig. 9.

Machine calculation of the complex cepstrum of Fig. 7.

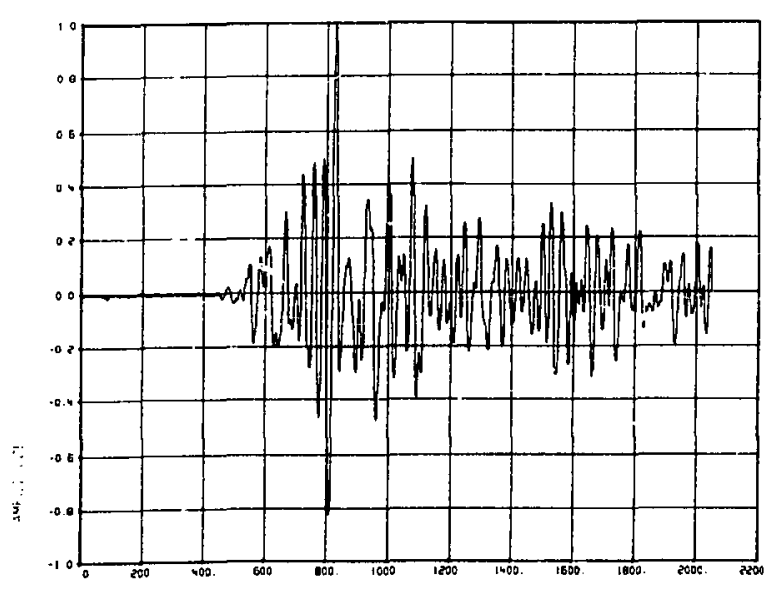

Fig. 11.

First 2048 points of the $A B Q / S R 0$ short period record of the North China earthquake. $\Delta t=0.05 \mathrm{~s}$.

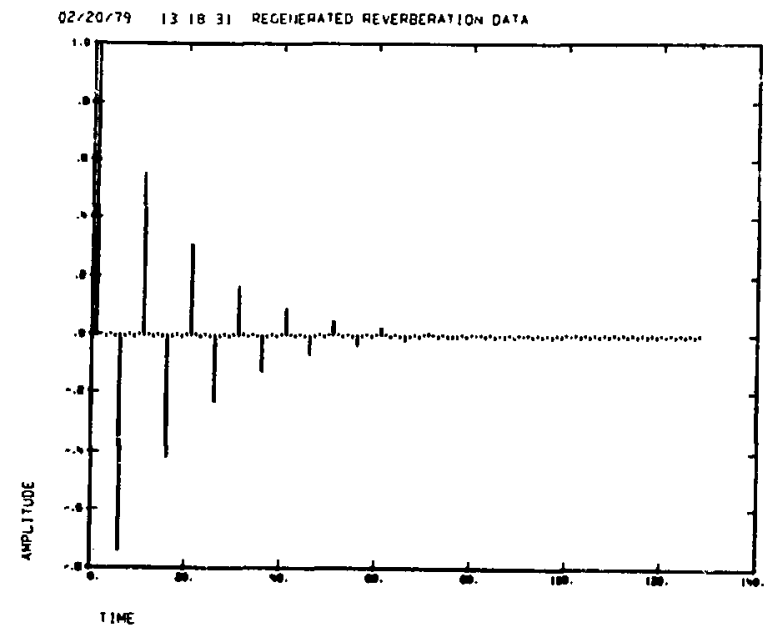

Fig. 10. Regeneration from Fig. 9.

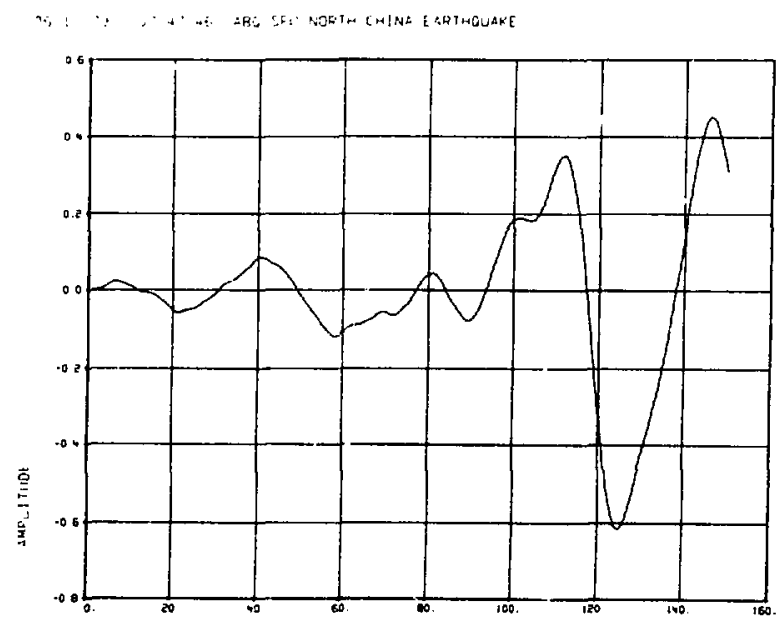

TIME

Fig. 12.

Detail of the beginning of the North China record of Fig. 11. 
series and the complex cepstrum computed. The region of maximum energy is shown in Fig. 13. The inverse operations were then applied to the complex cepstrum. The regenerated time series is displayed in Fig. 14 and may be compared with Fig. 12.

As another real world example, the seismic signal from the Nevada Test Site for the shot Farallones is displayed in Fig. 15. This was recorded at the Bolivian ASRO Station ZOBO. The approximate measured time of arrival was $11 \mathrm{~m}$, $13 \mathrm{~s}$. The estimated travel time from tables ${ }^{13}$ was $11 \mathrm{~m}, 12 \mathrm{~s}$ for the shortperiod body waves. The initial signal is shown in more detail in Fig. 16. The time series was then exponentially weighted with $a=0.945$ and the complex cepstrum computed. The result is shown in Fig. 17. The regenerated time series is shown in Fig. 18 and may be compared with Fig. 16. The cepstrum of Fig. 17 is low time filtered by setting all data above $T=10$ to zero and the inverse operations performed. The result shown in Fig. 19 is obtained. This is a crude estimate of the source wavelet.

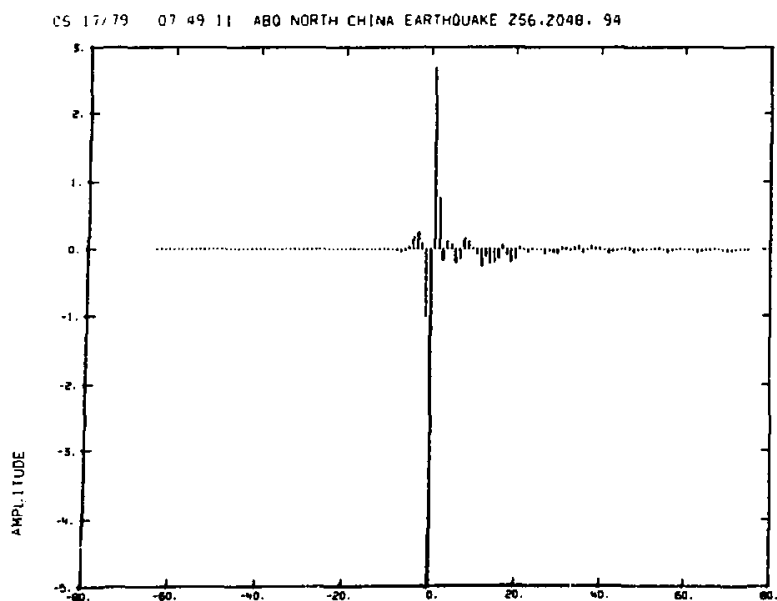

Fig. 13.

Portion of the machine calculation of the complex cepstrum of the North China se ismogram.

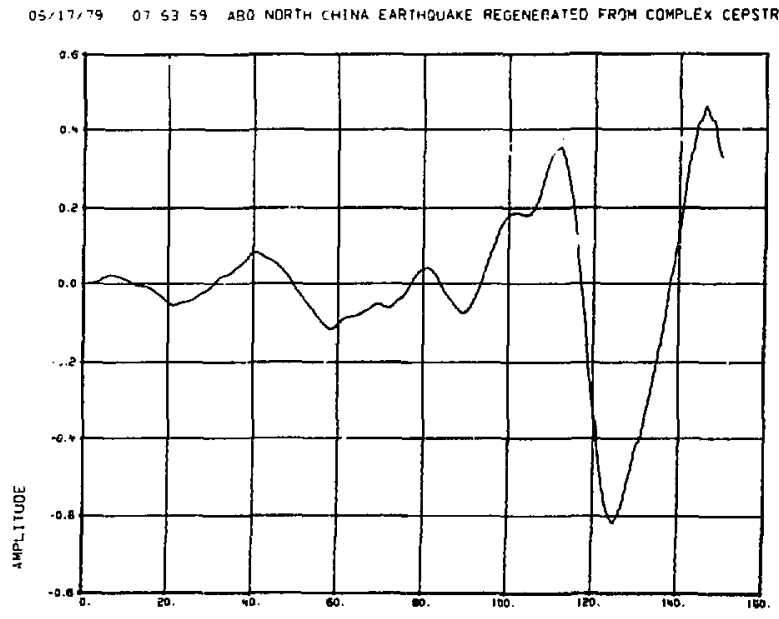

TIME

Fig. 14. Regeneration for the North China seismogram. 


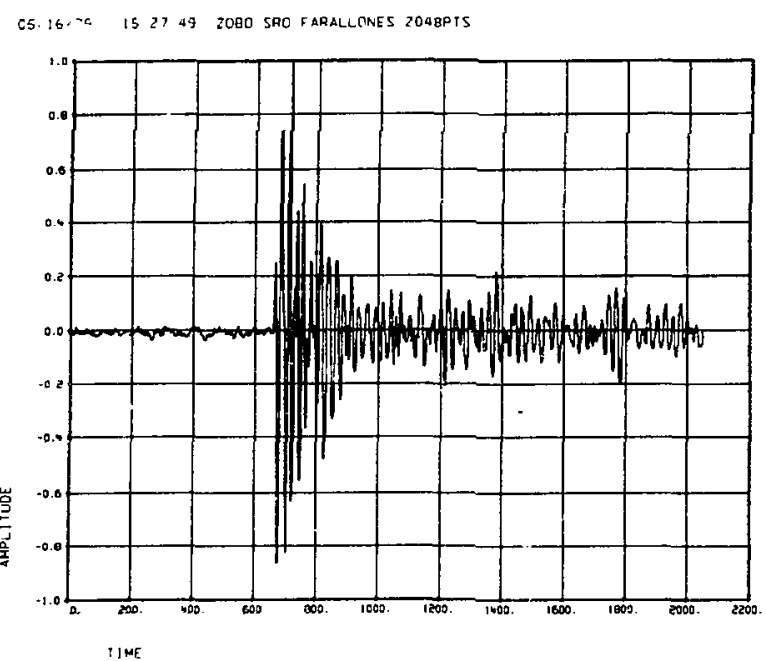

Fig. 15.

First 2048 points of the ZOBO/ASRO short period record of NTS shot Farallones. $\Delta t=0.05 \mathrm{~s}$.

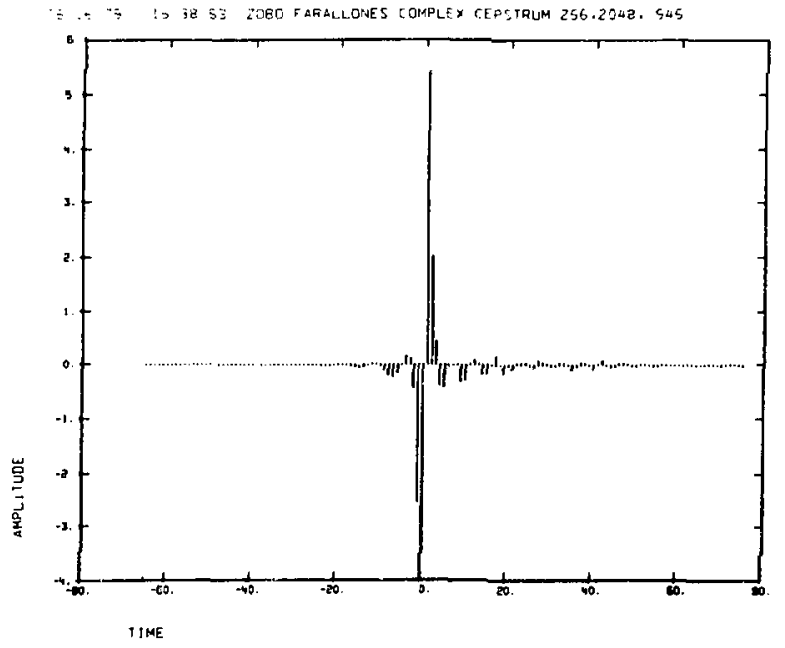

Fig. 17.

Portion of the machine calculation of the complex cepstrum of Fig. 16.

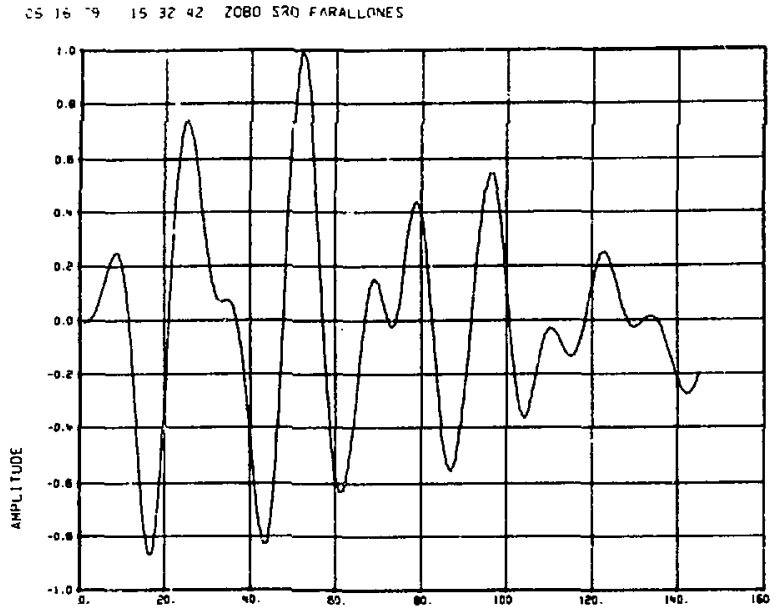

IIME

Fig. 16.

Detail of the beginning of the Farallones record.

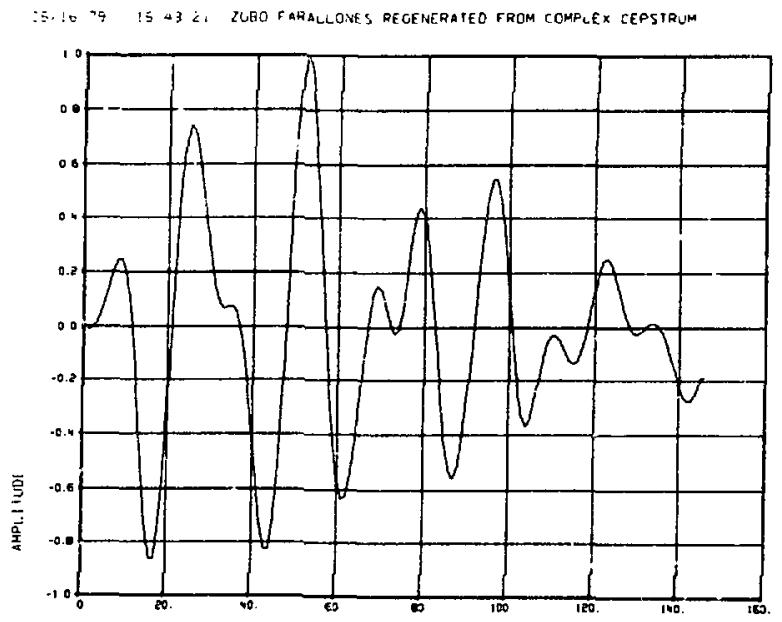

I I 19

Fig. 18.

Regeneration of the Farallones seismogram. 


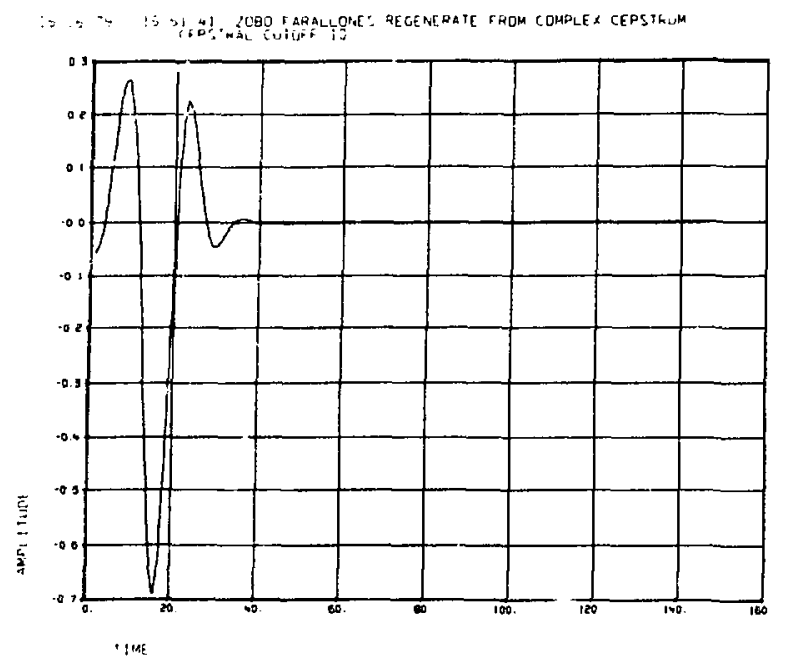

Fig. 19.

Crude estimate of the source wavelet by regenerating from a low time-gateá complex cepstrum.

\section{CONCLUSIONS}

All of the examples presented in this tutorial are for illustrative purposes only. They do suggest, however, that the present code might be useful in generating gated cepstra whose applicability to the pattern recognition problem $^{14,15}$ could be explored.

The problem of signal conditioning, apart from exponential weighting, was not considered. These are several aspects of this problem to be studied. Moreover, it is probably desirable to identify and use a more robust technique for computing the complex cepstrum than that used here.

\section{ACKNOWLEDGMENTS}

I. am indebted to John Hoffman and Jon Peterson of Albuquerque SRO for data and helpful discussions. Johr: Woolson, Teledyne Geotech, also provided data for this work. 


\section{REFERENCES}

1. G. C. Werth, R. F. Herbst, and D. L. Springer, "Amplitudes of Seismic Arrivals from the M Discontinuity," J. Geophys. Rsch. 67, 1587 (1962).

2. G. C. Werth and R. F. Herbst, "Curoarison of Amplitudes of Seismic Waves from Nuclear Explosions in Four Mediums," J. Geophys. Rsch. 68, 1463 (1963).

3. A. J. Chabai, "On Scaling of Craters Produced by Buried Explosives," J. Geophys. Rsch. 70, 5075 (1965).

4. R. Bracewe11, The Fourier Transform and its Applications (HcGraw-Hill, New York, 1965).

5. A. V. Oppenheim and R. W. Schafer, Digital Signal Processing (Prentice-Hall, Englewood Cliffs, New Jersey, 1975).

6. B. P. Bogert, M. J. Healy, and J. W. Tukey, The Quefrency Analys is of Time Series for Echoes: Cepstrum, Pseudo-Autocovariance, Cross Cepstrum and Saphe Cracking (M. Rosenblatt, Ed., Time Series Analysis, John Wiley, New York, 1963).

7. R. W. Schafer, "Echo Removal by Discrete Generalized Linear Filtering," MIT Technical Report 466, 1969.

8. J. M. Tribolet, "A New Phase Unwrapping Algorithm," IEEE Trans. Acoust., Speech and Signal Process. 25, 170 (1977).

9. T. J. Ulrych, "Applications of Homomorphic Deconvolution," Gecphys. 36 , 650 (1971).

10. T. J. Ulrych, 0. G. Jensen, R. M. Ellis, and P. G. Somerville, "Homomorphic Deconvolution of Some Teleseismic Events," Bu11. Seis. Soc. Amer. 62, $1269(1972)$.

11. W. H. Bakun and L. R. Johnson, "The Deconvolution of Teleseismic P Waves from Explosions Milrow and Cannikin," Geophys. J. R. Astr. Soc. 34, 321 (1973).

12. P. L. Stoffa, P. Buhl, and G. M. Bryan, "The Application of Homomorphic Deconvolution to Shallow-Water Marine Seismology - Part 1: Models," Geophys. 39, 401 (1974).

13. E. Herrin, "Introduction to 1968 Seismological Tables for P Phases," Bul1. Seism. Soc. Amer. 58, 1193 (1968).

14. J. E. Brolley, "Preprocessing of Seismic Signals for Pattern Recognition," Proc. of the NATO Advanced Study Inst. on Pattern Recognition and Sig. Processing, E.N.S.T. Paris, France, C. H. Chen, Ed., Sijthoff and Noordhoff, Alphen aan den Rijh, 1978.

15. J. E. Brolley, "Preprocessing of Seismic Signals by Haar Decomposition and Adaptive Filtering for Pattern Recognition," Los Alamos Scientific Laboratory report LA-7541-MS (November 1978). 